\title{
On metrizability and normability of 2-normed spaces
}

\author{
Anirban Kundu ${ }^{1}\left(\mathbb{D} \cdot \mathrm{T}_{\mathrm{Bag}}{ }^{1} \cdot \mathrm{Sk} . \mathrm{Nazmul}^{2}\right.$
}

Received: 20 May 2018 / Accepted: 5 March 2019 / Published online: 14 March 2019

(c) The Author(s) 2019

\begin{abstract}
In this present work, the topological structure of 2-normed spaces is studied from the viewpoint of topological vector spaces. A separating family of seminorms is induced from a given 2-norm, and the criteria for metrizability and normability of 2-normed spaces are investigated using this family. A sufficient condition for metrizability and a necessary and sufficient condition for normability of 2-normed spaces are deduced during this investigation.
\end{abstract}

Keywords 2-Normed space $\cdot$ Topological vector space $\cdot$ Separating family of seminorms

Mathematics Subject Classification 46A19 · 46A99 · 46B20 · 46B99

\section{Introduction}

The history of functional analysis is not very old. The idea grew up in early twentieth century. Researchers felt the necessity of this subject during the studies of integration theory and integral equations. However, Banach was the pioneer of formal functional analysis. In 1922, he defined normed linear spaces as a set of axioms. Since then, mathematicians keep on trying to find a proper generalization of this concept. The first notable attempt was by Vulich [10]. He introduced $\mathbf{K}$-normed space in 1937. In another process of generalization, Gähler [1] introduced 2-metric in 1963. As a continuation of his research, Gähler [2] proposed a mathematical structure, called 2-normed space, as a generalization of normed linear spaces. As a further extension, he introduced $n$-metric and $n$-norm in his subsequent works [3-5] and regarded normed linear spaces as 1-normed spaces. However, many researchers disagree to consider 2-norm

Anirban Kundu

anirbankundu92@gmail.com

T. Bag

tarapadavb@gmail.com

Sk. Nazmul

sk.nazmul_math@yahoo.in

1 Department of Mathematics, Siksha-Bhavana, VisvaBharati, Santiniketan, Birbhum, West-Bengal 731235, India

2 Department of Mathematics, Bankura University, Bankura, West-Bengal 722155, India and $n$-norm as generalization of norm. In spite of this disagreement, several researchers have worked on this topic for decades. They have found out many interesting properties of this space and lots of fixed point theorems are established.

In this article, we have studied 2-normed spaces from the viewpoint of topological vector spaces. A separating family of seminorms is induced from the 2-norm and used it as a one-way bridge between 2-normed spaces and topological vector spaces. Our method gives an alternative proof of the statement that every 2-normed space is a locally convex topological vector space. The line of proof gives more insight of the space and helps to find a sufficient condition for metrizability. Additionally, we have been able to find out a necessary and sufficient condition for normability of a 2-normed space. As a complementary result, a norm is derived for such normable 2-normed space. We also have shown that one can derive a norm from a given 2-norm in such a way that convergence of sequences for both 2-norm and the derived norm are equivalent if and only if the 2-norm is normable. This answers an open problem given by Gunawan assnd Mashadi [7].

The rest of this article is divided into four different sections. Section "Preliminaries" deals with the definitions and results available in the literature which are used in the subsequent sections of this article. The topology of 2-normed spaces is studied from the viewpoint of topological vector spaces in Section "Topology of 2-normed spaces". A sufficient condition for metrizability is also derived in this section. Section "Normability of 2-normed spaces" solely deals 
with the normability problem of 2-normed spaces. A necessary and sufficient condition for normability of 2-normed spaces is established in this section. A possible construction of a norm for normable 2-normed spaces is given, and an open problem by Gunawan and Mashadi [7] is answered in Section "Construction of a norm for a normable 2-normed space".

\section{Preliminaries}

Definition 1 [1] Let $X$ be a non-empty set. A function

$\sigma: X \times X \times X \rightarrow \mathbb{R}_{\geq 0}$

is called a 2-metric on $X$, if $\sigma$ satisfies the following:

(I) For any two elements $a$ and $b$ in $X$, there exists $c \in X$ such that $\sigma(a, b, c) \neq 0$,

(II) $\sigma(a, b, c)=0$ if at least two out of three elements $a, b, c$ are same,

(III) $\sigma(a, b, c)=\sigma(a, c, b)=\sigma(b, c, a)$ where $a, b, c \in X$,

(IV) $\sigma(a, b, c) \leq \sigma(a, b, d)+\sigma(a, d, c)+\sigma(d, b, c)$ for all $a, b, c, d \in X$.

The pair $(X, \sigma)$ is called a 2-metric space.

Definition 2 [1] A 2-metric space $(X, \sigma)$ is said to satisfy property $(K)$ if the following holds:

For a sequence of points $a, a_{1}, a_{2}, \ldots, a_{n}, \ldots$ of $X$ if there exist $b, c \in X$ satisfying

(i) $\sigma(a, b, c) \neq 0$,

(ii) $\lim _{n \rightarrow \infty} \sigma\left(a, b, a_{n}\right)=0$,

(iii) $\lim _{n \rightarrow \infty} \sigma\left(a, c, a_{n}\right)=0$,

then $\lim _{n \rightarrow \infty} \sigma\left(a, a^{\prime}, a_{n}\right)=0$ for all $a^{\prime} \in X$.

Gähler also studied the topology of 2-metric spaces. For any two points $a, b \in X$ and any $\epsilon>0$, he introduced the set

$U_{\epsilon}(a, b):=\{c \in X: \sigma(a, b, c)<\epsilon\}$

and called it as $\epsilon$-neighbourhood of $a$ and $b$. The smallest topology containing the set

$\mathcal{U}:=\left\{U_{\epsilon}(a, b): a, b \in X, \epsilon>0\right\}$

is called the natural topology or the topology generated by 2-metric in $X$. The set of all possible finite intersections of such neighbourhoods forms a base for this topology. Gähler considered this as the topology of 2-metric space.

As a consequence of 2-metric, he introduced 2-norm.

Definition 3 [2] Let $X$ be a vector space over the field $\mathbb{R}$ with dimension $\geq 2$. A function $\|\cdot, \cdot\|: X \times X \rightarrow \mathbb{R}_{\geq 0}$

is called a 2-norm over $X$ if:

(I) $\|x, y\| \geq 0$ for every $x, y \in X,\|x, y\|=0$ if and only if $x$ and $y$ are linearly dependent,

(II) $\|x, y\|=\|y, x\|$ for every $x, y \in X$,

(III) $\|\alpha x, y\|=|\alpha|\|x, y\|$ for every $x, y \in X$ and $\alpha \in \mathbb{R}$,

(IV) $\|x, y+z\| \leq\|x, y\|+\|x, z\|$ for every $x, y, z \in X$.

The pair $(X,\|\cdot, \cdot\|)$ is called a 2-normed space.

Theorem 1 [2] Let $(X,\|\cdot, \cdot\|)$ be a 2-normed space. Define a function $\sigma$ by

$\sigma(a, b, c):=\|a-c, b-c\|$

for all $a, b, c \in X$. Then $(X, \sigma)$ forms a 2-metric space.

So a 2-normed space can be treated as a 2-metric space with the induced 2-metric $\sigma$ defined in Theorem 1. The topology of a 2-normed space is defined as the topology of the 2-metric space induced by that 2-norm. Moreover, if $\sigma$ satisfies property $(K)$, then it is said that the 2-norm satisfies property $(K)$.

Metrizability and normability of 2-normed spaces are two interesting areas of study. Here is an example related to this topic.

Example 1 [2] Consider the set of all real-valued functions on $I=(0, \infty) \times(0, \infty)$, denoted by $\mathcal{F}(I, \mathbb{R})$. For each $i=\left(i_{1}, i_{2}\right) \in I$, let $e_{i} \in \mathcal{F}(I, \mathbb{R})$ be defined as

$e_{i}(x)=\left\{\begin{array}{l}1 \text { if } x=i \\ 0 \text { otherwise. }\end{array}\right.$

The set $L=\left\{e_{i}: i \in I\right\}$ is linearly independent. Choose the subspace $X$ spanned by $L$. Gähler defined a 2-norm on $X$ in two steps. At first, he defined the following

$\left\|e_{i}, e_{j}\right\|:=\left\{\begin{array}{lll}0 & \text { if } \quad i=j \\ \sup \left\{i_{2}, j_{2}\right\} & \text { if } \quad i_{1}=j_{1} \text { and } i_{2} \neq j_{2} \\ 1 & \text { if } \quad i_{1} \neq j_{1},\end{array}\right.$

where $i=\left(i_{1}, i_{2}\right)$ and $j=\left(j_{1}, j_{2}\right)$ are elements of $I$.

Now, for any two elements $x, y \in X$ there is a finite subset $I^{\prime}$ of $I$ such that

$x=\sum_{i \in I^{\prime}} c_{i} e_{i}$

and

$y=\sum_{i \in I^{\prime}} d_{i} e_{i}$. 
Using this fact, Gähler defined a 2-norm on $X$ as follows:

$\|x, y\|:=\frac{1}{2} \sum_{i, j \in I^{\prime}}\left|c_{i} d_{j}-d_{i} c_{j}\right|\left\|e_{i}, e_{j}\right\|$.

Gähler showed that this 2-normed space is not metrizable. In particular, it is not normable.

Here is a result related to the condition of normability of 2-normed spaces.

Theorem 2 [2] A 2-normed space $(X,\|\cdot, \cdot\|)$ has property $(K)$ if and only if for all $a^{*}, b^{*} \in X$ with $\left\|a^{*}, b^{*}\right\| \neq 0$ the norm on $X$ defined by

$\|a\|:=\left\|a^{*}, a\right\|+\left\|b^{*}, a\right\|$ for all $a \in X$

has the same topology as $\|\cdot \cdot \cdot\|$.

However, Theorem 2 does not provide a complete answer to the question of normability of 2-normed spaces. Section 4 of this paper gives a proper answer to this question.

A definition of Cauchy sequence for $n$-normed spaces is given in [7]. We rewrite this for 2-normed spaces as follows:

Definition 4 A sequence $\left\{x_{n}\right\}$ in a 2-normed space $(X,\|\cdot, \cdot\|)$ is called a Cauchy sequence if for every $y \in X$ the following holds: $\lim _{m, n \rightarrow \infty}\left\|x_{n}-x_{m}, y\right\|=0$.

Definition 5 [6] A sequence $\left\{x_{n}\right\}$ in a 2-normed space $(X,\|\cdot, \cdot\|)$ is said to converge to $x \in X$ if $\lim _{n \rightarrow \infty}\left\|x_{n}-x, y\right\|=0$ for all $y \in X$.

Definition 6 [11] A topology $\tau$ on a vector space $X$ over the field $\mathbb{K}$ (where $\mathbb{K}=\mathbb{R}$ or $\mathbb{C}$ ) is called a vector topology if the following two conditions hold:

(a) $\tau$ is Hausdorff,

(b) the vector space operations are continuous with respect to $\tau$.

The space $(X, \tau)$ is called a topological vector space.

Remark 1 Instead of considering the Hausdorff property of $\tau$, Rudin [9] prefers to consider ( $a$ ) to be "Every point of $X$ is a closed set". However, both the definitions are equivalent in nature.

Definition 7 [11] Let $X$ be a topological vector space. A set $C \subseteq X$ is called convex if

$t C+(1-t) C \subseteq C$

where $0 \leq t \leq 1$.
Definition 8 [11] A subset $B$ of $X$ is said to be balanced if $\alpha B \subseteq B$ whenever $\alpha \in \mathbb{K}$ such that $|\alpha| \leq 1$.

Definition 9 [11] A topological vector space $(X, \tau)$ is called locally convex if it has a local base at 0 consisting convex balanced sets.

Definition 10 [9] A set $B \subseteq X$ is said to be bounded if for every open set $U$ containing 0 in $X$ there exists $s>0$ such that for all $t>s$ we have $B \subseteq t U$.

Remark 2 In Definition 10, one can use the phrase " $t \geq s$ " instead of " $t>s$ " since both cases are equivalent in nature.

Definition 11 [9] A topological vector space is said to be locally bounded if $X$ has a bounded open set containing 0 .

Definition 12 [9] Let $X$ be a vector space over the field $\mathbb{K}$. A function $p: X \rightarrow \mathbb{R}$ is called a seminorm on $X$ if it satisfies:

(a) $p(x+y) \leq p(x)+p(y)$

(b) $p(\alpha x)=|\alpha| p(x)$

for all $x, y \in X$ and $\alpha \in \mathbb{K}$.

Definition 13 [9] A family of seminorms

$\mathscr{P}=\left\{p_{\alpha}: \alpha \in \Lambda\right\}$

is called a separating family if for each $x \in X$ there exists $p_{\alpha} \in \mathscr{P}$ such that $p_{\alpha}(x) \neq 0$.

Definition 14 [8] Let $X$ be a vector space and

$\mathscr{P}=\left\{p_{\alpha}: \alpha \in \Lambda\right\}$

be a family of seminorms on $X$. The $\alpha$ th open strip of radius $r>0$ and centred at $x \in X$ is defined by:

$B_{r}^{\alpha}(x):=\left\{y \in X: p_{\alpha}(x-y)<r\right\}$, where $\alpha \in \Lambda$.

Definition 15 [8] Let $X$ be a vector space and

$\mathscr{P}=\left\{p_{\alpha}: \alpha \in \Lambda\right\}$

be a family of seminorms. Let $\mathscr{E}$ be the collection of all open strips in $X$, i.e.

$\mathscr{E}:=\left\{B_{r}^{\alpha}(x): x \in X, r>0, \alpha \in \Lambda\right\}$.

The topology, $\tau(\mathscr{E})$, generated by $\mathscr{E}$ is called the topology induced by the family of seminorms $\mathscr{P}$. Throughout this paper, we have denoted this topology by $\tau_{\mathscr{P}}$.

Remark 3 The set, $\mathcal{B}$, of all finite intersections of the elements of $\mathscr{E}$ forms a base for the topology $\tau_{\mathscr{P}}$. 
Proposition 1 [8] Vector addition and scalar multiplication are continuous for the topology $\tau_{\mathscr{P} \text {. }}$

Proposition 2 [8] $\tau_{\mathscr{P}}$ is Hausdorff if and only if $\mathscr{P}$ is a separating family of seminorms.

Remark 4 Using Propositions 1 and 2, it can be concluded that $\tau_{\mathscr{P}}$ is a topological vector space if and only if $\mathscr{P}$ is a separating family of seminorms.

Proposition 3 [11] If $\mathscr{P}$ is a separating family of seminorms, then $\tau_{\mathscr{P}}$ is a locally convex topological vector space.

Theorem 3 [9] A topological vector space is metrizable if and only if it has a countable local base.

Theorem 4 [9] A topological vector space is normable if and only if there exists a convex bounded open set containing origin.

\section{Topology of 2-normed spaces}

In this section, a separating family of seminorms have been constructed from a 2-norm. As a consequence, a sufficient condition for metrizability is derived.

Proposition 4 Consider a 2-normed space $(X,\|\cdot, \cdot\|)$ and $\mathscr{B}$ be a Hamel basis of $X$. Then, the family

$\mathscr{P}:=\left\{p_{a}: a \in \mathscr{B}\right\}$

forms a separating family of seminorms on $X$, where for every $a \in \mathscr{B}$

$p_{a}: X \rightarrow \mathbb{R}$

is defined by $p_{a}(x)=\| x$, a $\|$ for all $x \in X$.

Proof Fix any $a \in \mathscr{B}$. We have:

(a) For any $x, y \in X$,

$$
\begin{aligned}
p_{a}(x+y) & =\|x+y, a\| \\
& \leq\|x, a\|+\|y, a\| \\
& =p_{a}(x)+p_{a}(y) .
\end{aligned}
$$

(b) For any $x \in X$ and $\alpha \in \mathbb{K}$,

$$
\begin{aligned}
p_{a}(\alpha x) & =\|\alpha x, a\| \\
& =|\alpha|\|x, a\| \\
& =|\alpha| p_{a}(x) .
\end{aligned}
$$

Hence, $p_{a}$ is a seminorm for each $a \in \mathscr{B}$ and so

$\mathscr{P}=\left\{p_{a}: a \in \mathscr{B}\right\}$

forms a family of seminorms on $X$. Now, for any $x \in X$, there exists $a \in \mathscr{B}$ such that $x$ and $a$ are linearly independent. Thus, $\|x, a\| \neq 0$ and hence $p_{a}(x) \neq 0$. Therefore, $\mathscr{P}$ forms a separating family of seminorms.

Our next theorem leads to the conclusion that a 2-normed space is a topological vector space. This was already done by Gähler in [2]. But, here we prove it in a different way. Our method is more constructive in nature. It helps us to find out a necessary and sufficient condition for normability which is discussed in Sect. 4 of this article.

Theorem 5 Let $(X,\|\cdot, \cdot\|)$ be a 2-normed space. Then, the topology of $(X,\|\cdot, \cdot\|)$ coincides with the topological vector space induced by the separating family of seminorms $\mathscr{P}$.

Proof Let $\sigma$ be the 2-metric induced by $\|\cdot, \cdot\|$. Take any $a, b \in X$ and $\epsilon>0$. Then, the $\epsilon$ neighbourhood of $a$ and $b$ is,

$$
\begin{aligned}
U_{\epsilon}(a, b) & :=\{x \in X: \sigma(a, b, x)<\epsilon\} \\
& =\{x \in X: \sigma(b, x, a)<\epsilon\} \\
& =\{x \in X:\|b-a, x-a\|<\epsilon\} \\
& =\{x \in X:\|a-x, b-a\|<\epsilon\} .
\end{aligned}
$$

$\mathscr{P}=\left\{p_{a}: a \in \mathscr{B}\right\}$ is a separating family of seminorms where $\mathscr{B}$ is a Hamel basis of $X$. As the first step, let us prove that

$\mathscr{E}:=\left\{B_{r}^{a}(x): x \in X, r>0, a \in \mathscr{B}\right\}$

is a subset of

$\mathcal{U}:=\left\{U_{\epsilon}(a, b): a, b \in X, \epsilon>0\right\}$.

Consider any element $B_{r}^{a}(x)$ from $\mathscr{E}$. Then,

$$
\begin{aligned}
B_{r}^{a}(x) & =\left\{y \in X: p_{a}(x-y)<r\right\} \\
& =\{y \in X:\|x-y, a\|<r\} \\
& =\{y \in X:\|x-y, a+x-x\|<r\} \\
& =U_{r}(x, a+x) .
\end{aligned}
$$

Hence, $B_{r}^{a}(x) \in \mathscr{U}$ and so $\mathscr{E} \subseteq \mathscr{U}$.

Therefore, $\tau_{\mathscr{P}}$ is a subset of the natural topology of the 2-normed space $(X,\|\cdot, \cdot\|)$. U.

To prove the converse part, take any element $U_{\epsilon}(a, b)$ from

Consider any $x \in U_{\epsilon}(a, b)$. Then, we have 
$\|a-x, b-a\|<\epsilon$.

Let us say that $\|a-x, b-a\|=\epsilon^{\prime}$. So $\epsilon-\epsilon^{\prime}>0$.

Now, there exist $a_{1}, a_{2}, \ldots, a_{n} \in \mathscr{B}$ and $c_{1}, c_{2}, \ldots, c_{n} \in \mathbb{K}$ such that

$b-a=c_{1} a_{1}+c_{2} a_{2}+\cdots+c_{n} a_{n}$.

Let

$\delta:=\frac{\epsilon-\epsilon^{\prime}}{\left|c_{1}\right|+\left|c_{2}\right|+\cdots+\left|c_{n}\right|}$.

Then, for any $y \in \bigcap_{i=1}^{n} B_{\delta}^{a_{i}}(x)$ we obtain

$$
\begin{aligned}
& p_{a_{i}}(x-y)<\delta \text { for all } i=1,2, \ldots, n \\
& \quad \Rightarrow\left\|x-y, a_{i}\right\|<\delta \text { for all } i=1,2, \ldots, n \\
& \quad \Rightarrow\|x-y, b-a\| \leq \sum_{i=1}^{n}\left|c_{i}\right| \cdot\left\|x-y, a_{i}\right\|<\delta \cdot \sum_{i=1}^{n}\left|c_{i}\right| \\
& \quad \Rightarrow\|x-y, b-a\|<\epsilon-\epsilon^{\prime} .
\end{aligned}
$$

So, we have

$$
\begin{aligned}
\|a-y, b-a\| & \leq\|a-x, b-a\|+\|x-y, b-a\| \\
& <\epsilon^{\prime}+\left(\epsilon-\epsilon^{\prime}\right) \\
& =\epsilon .
\end{aligned}
$$

Hence, $y \in U_{\epsilon}(a, b)$ and so $x \in \bigcap_{i=1}^{n} B_{\delta}^{a_{i}}(x) \subseteq U_{\epsilon}(a, b)$. This concludes that $U_{\epsilon}(a, b)$ is an open set in $\left(X, \tau_{\mathscr{P}}\right)$.

Therefore, $\mathcal{U} \subseteq \tau_{\mathscr{P}}$. Thus, the smallest topology containing the elements of $\mathcal{U}$, which is the natural topology of the 2-normed space, is a subset of $\tau_{\mathscr{P}}$.

Thus, $\tau_{\mathscr{P}}$ coincides with the natural topology of $(X,\|\cdot, \cdot\|)$.

Remark 5 As a concluding note, we can say that

$\mathcal{B}:=\left\{\bigcap_{i=1}^{n} B_{r_{i}}^{a_{i}}\left(x_{i}\right): x_{i} \in X, a_{i} \in \mathscr{B}, r_{i}>0, n \in \mathbb{N}\right\}$,

i.e. the set of all possible finite intersections of the elements of $\mathscr{E}$, is a base for the natural topology of $(X,\|\cdot, \cdot\|)$.

Proposition 5 If for $a \in \mathscr{B}$ and $n \in \mathbb{N}$ we define

$V(a, n):=B_{\frac{1}{n}}^{a}(0)$,

then
$\mathcal{B}_{0}:=\left\{\bigcap_{i=1}^{k} V\left(a_{i}, n_{i}\right): a_{i} \in \mathscr{B}, n_{i} \in \mathbb{N}, k \in \mathbb{N}\right\}$

is a local base at 0 .

Proof Let $G$ be an open set containing 0 . Then, there exists $\bigcap_{i=1}^{k} B_{r_{i}}^{a_{i}}\left(x_{i}\right) \in \mathscr{B}$ such that

$0 \in \bigcap_{i=1}^{k} B_{r_{i}}^{a_{i}}\left(x_{i}\right) \subseteq G$.

Now for $i=1,2, \ldots, k$, we have $0 \in B_{r_{i}}^{a_{i}}\left(x_{i}\right)$ and so

$r_{i}^{\prime}:=\left\|x_{i}, a_{i}\right\|<r_{i}$.

Choose $n_{i} \in \mathbb{N}$ such that $\frac{1}{n_{i}}<r_{i}-r_{i}^{\prime}$. Then, we have

$$
\begin{aligned}
y \in V\left(a_{i}, n_{i}\right) & \Rightarrow y \in B_{\frac{1}{n_{i}}}^{a_{i}}(0) \\
& \Rightarrow\left\|y, a_{i}\right\|<\frac{1}{n_{i}} \\
& \Rightarrow\left\|y-x_{i}, a_{i}\right\| \leq\left\|y, a_{i}\right\|+\left\|x_{i}, a_{i}\right\|<\frac{1}{n_{i}}+r_{i}^{\prime} \\
& \Rightarrow\left\|y-x_{i}, a_{i}\right\|<r_{i} \\
& \Rightarrow y \in B_{r_{i}}^{a_{i}}\left(x_{i}\right) .
\end{aligned}
$$

Hence, $0 \in V\left(a_{i}, n_{i}\right) \subseteq B_{r_{i}}^{a_{i}}\left(x_{i}\right)$ for each $i=1,2, \ldots, k$. Therefore,

$0 \in \bigcap_{i=1}^{k} V\left(a_{i}, n_{i}\right) \subseteq \bigcap_{i=1}^{k} B_{r_{i}}^{a_{i}}\left(x_{i}\right) \subseteq G$.

Thus, $\mathcal{B}_{0}$ is a local base at 0 .

We prove the main theorem of this section using Proposition 5 as follows:

Theorem 6 A 2-normed space with a countable Hamel basis is metrizable.

Proof Let $(X,\|\cdot, \cdot\|)$ be a 2-normed space with a countable Hamel basis $\mathscr{B}$. Then, the set $\mathcal{B}_{0}$ defined in Proposition 5 is countable. Hence, the topology of $(X,\|\cdot, \cdot\|)$ has a countable local base, and so, by Theorem $3,(X,\|\cdot, \cdot\|)$ is metrizable.

Converse of Theorem 6 may not be true. It is briefly explained in Example 2. 


\section{Normability of 2-normed spaces}

In this section, we have developed a theory to find a necessary and sufficient condition for normability of 2-normed spaces. We start with the following definition:

Definition 16 A 2-normed space $(X,\|\cdot, \cdot\|)$ is said to satisfy property $\left(K_{m}\right)$ for some $m \in \mathbb{N}$ if there exist linearly independent elements $a_{1}, a_{2}, \ldots, a_{m}$ in $X$ such that for every sequence of points $x, x_{1}, x_{2}, x_{3}, \ldots$ of $X$,

$\lim _{n \rightarrow \infty}\left\|x-x_{n}, a_{i}\right\|=0$ for all $i=1,2, \ldots, m$

implies $\lim _{n \rightarrow \infty}\left\|x-x_{n}, a\right\|=0$ for all $a \in X$, i.e. $x_{n} \rightarrow x$ in the 2-normed space $X$.

Remark 6 Every finite-dimensional 2-normed space with dimension $m \geq 2$ satisfies the property $\left(K_{m}\right)$.

Theorem 7 Let $(X,\|\cdot, \cdot\|)$ be a 2-normed space. If this space satisfies the property $\left(K_{m}\right)$ for some $m \in \mathbb{N}$, then it is normable.

Proof Given that $(X,\|\cdot, \cdot\|)$ satisfies the property $\left(K_{m}\right)$ for some $m \in \mathbb{N}$, so there exist $a_{1}, a_{2}, \ldots, a_{m} \in X$, which are linearly independent, such that for every sequence of points $x, x_{1}, x_{2}, x_{3}, \ldots$ of $X$,

$\lim _{n \rightarrow \infty}\left\|x-x_{n}, a_{i}\right\|=0$ for all $i=1,2, \ldots, m$

implies $\lim _{n \rightarrow \infty}\left\|x-x_{n}, a\right\|=0$ for all $a \in X$.

There exists a Hamel basis $\mathscr{B}$ of $X$ such that $\left\{a_{1}, a_{2}, \ldots, a_{m}\right\}$ is a subset of $\mathscr{B}$. From Proposition 4 and Theorem 5 , we can conclude that the topology of $(X,\|\cdot, \cdot\|)$ coincides with the locally convex topological vector space generated by the separating family of seminorms

$\mathscr{P}:=\left\{p_{a}: a \in \mathscr{B}\right\}$

on $X$, where for every $a \in \mathscr{B}$

$p_{a}: X \rightarrow \mathbb{R}$

is defined by $p_{a}(x)=\|x, a\|$ for all $x \in X$.

We claim that, for each $a \in \mathscr{B}$ and $\epsilon>0$, there exists $\delta>0$ such that

$\bigcap_{i=1}^{m} B_{\delta}^{a_{i}}(0) \subseteq B_{\epsilon}^{a}(0)$

If not, then there exists $a_{0} \in \mathscr{B}$ and $\epsilon_{0}>0$ for which no such $\delta$ exists. So for each $\delta=\frac{1}{k}$ where $k \in \mathbb{N}$, there exists

$x_{k} \in \bigcap_{i=1}^{m} B_{\delta}^{a_{i}}(0) \backslash B_{\epsilon_{0}}^{a_{0}}(0)$.
Therefore, for all $n \in \mathbb{N},\left\|x_{n}, a_{0}\right\| \geq \epsilon_{0}$. On the other hand, $\lim _{n \rightarrow \infty}\left\|x_{n}, a_{i}\right\|=0$ for each $i=1,2, \ldots, m$. This is a contradiction to our hypothesis. So Eq. (1) holds.

Now, $B_{\delta}^{a_{i}}(0)=\delta B_{1}^{a_{i}}(0)$. So Eq. (1) transforms into

$\delta\left(\bigcap_{i=1}^{m} B_{1}^{a_{i}}(0)\right) \subseteq B_{\epsilon}^{a}(0)$

and so

$\bigcap_{i=1}^{m} B_{1}^{a_{i}}(0) \subseteq \frac{1}{\delta} B_{\epsilon}^{a}(0)$

Take $s=\frac{1}{\delta}$. Then, for each $t \geq s$, we have

$\bigcap_{i=1}^{m} B_{1}^{a_{i}}(0) \subseteq t B_{\epsilon}^{a}(0)$.

Now we are ready to prove that $B=\bigcap_{i=1}^{m} B_{1}^{a_{i}}(0)$ is bounded.

Consider any open set $G$ containing 0 . From Proposition 5 , we can say that

$\bigcap_{i=1}^{k} V\left(b_{i}, n_{i}\right) \subseteq G$

for some $b_{i} \in \mathscr{B}, n_{i}, k \in \mathbb{N}$. Now there exists $\epsilon>0$ such that $\epsilon<\frac{1}{n_{i}}$ for each $i=1,2, \ldots, k$. So

$\bigcap_{i=1}^{k} B_{\epsilon}^{b_{i}}(0) \subseteq \bigcap_{i=1}^{k} V\left(b_{i}, n_{i}\right) \subseteq G$

By Eq. (2), there exists $s_{i}>0$ such that

$\bigcap_{i=1}^{m} B_{1}^{a_{i}}(0) \subseteq t B_{\epsilon}^{b_{i}}(0)$

for all $t \geq s_{i}$.

Take $s=\sup \left\{s_{1}, s_{2}, \ldots, s_{k}\right\}$. Then, for all $t \geq s$, we have

$\bigcap_{i=1}^{m} B_{1}^{a_{i}}(0) \subseteq t\left(\bigcap_{i=1}^{k} B_{\epsilon}^{b_{i}}(0)\right)$

Using Eqs. (3) and (4), we get

$B=\bigcap_{i=1}^{m} B_{1}^{a_{i}}(0) \subseteq t G$

for all $t \geq s$. Hence, $B$ is a bounded open set containing 0 . On the other hand, $B$ is the intersection of some open strips, which are convex. So $B$ is convex.

Therefore, using Theorem 4 we can conclude that the topology of $(X,\|\cdot, \cdot\|)$ is normable.

Converse of Theorem 7 is also true. 
Theorem 8 A 2-normed space satisfies property $\left(K_{m}\right)$ for some $m \in \mathbb{N}$ if it is normable.

Proof Let $X$ be a 2-normed space, with Hamel basis $\mathscr{B}$, which is normable. Then, there exists a bounded open set $G$ containing 0. For some $a_{i} \in \mathscr{B}, n_{i}, m \in \mathbb{N}$,

$\bigcap_{i=1}^{m} V\left(a_{i}, n_{i}\right) \subseteq G$.

Now, for any $a \in \mathscr{B}$ and any $\epsilon>0$, the set $B_{\epsilon}^{a}(0)$ is an open set containing 0 . So there exists $s>0$ such that $G \subseteq t B_{\epsilon}^{a}(0)$ for all $t \geq s$. Therefore, for all $t \geq s$

$$
\begin{aligned}
& \bigcap_{i=1}^{m} V\left(a_{i}, n_{i}\right) \subseteq t B_{\epsilon}^{a}(0), \\
& \text { i.e. } \\
& \bigcap_{i=1}^{m} \frac{1}{t} V\left(a_{i}, n_{i}\right) \subseteq B_{\epsilon}^{a}(0) .
\end{aligned}
$$

In particular, for $t=s$, we get $\frac{1}{s} V\left(a_{i}, n_{i}\right)=B_{\frac{1}{n_{i} s}}^{a_{i}}(0)$ and so

$$
\bigcap_{i=1}^{m} B_{\frac{1}{n_{i} s}}^{a_{i}}(0) \subseteq B_{\epsilon}^{a}(0) .
$$

If $x$ is an element of $X$ and $\left\{x_{n}\right\}$ is a sequence in $X$ such that $\lim _{n \rightarrow \infty}\left\|x-x_{n}, a_{i}\right\|=0$ for all $i=1,2, \ldots, m$, then there exists

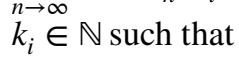

$$
\left\|x-x_{n}, a_{i}\right\|<\frac{1}{n_{i} s}
$$

for all $n \geq k_{i}$, where $i=1,2, \ldots, m$.

Take $k=\sup \left\{k_{1}, k_{2}, \ldots, k_{m}\right\}$. Then, for all $n \geq k$, we have $x-x_{n} \in \bigcap_{i=1}^{m} B_{\frac{1}{n_{i}}}^{a_{i}}(0) \subseteq B_{\epsilon}^{a}(0)$.

Therefore, $\left\|x-x_{n}, a\right\|<\epsilon$ for all $n \geq k$ and so we have $\lim _{n \rightarrow \infty}\left\|x-x_{n}, a\right\|=0$.

Since $a \in \mathscr{B}$ is arbitrary, this holds for all $a \in X$. Thus, $X$ satisfies property $\left(K_{m}\right)$.

From Theorems 7 and 8, we obtain a necessary and sufficient condition for normability given below.

Theorem 9 A 2-normed space is normable if and only if it satisfies the property $\left(K_{m}\right)$ for some $m \in \mathbb{N}$.

From Theorem 9 and Remark 6, we may conclude the following:
Corollary 1 A finite-dimensional 2-normed space is normable.

\section{Construction of a norm for a normable 2-normed space}

So far, a necessary and sufficient condition for normability of 2-normed spaces is discussed. In this section, we derive a norm from a normable 2-norm such that both have the same topology. We start with the following proposition.

Proposition 6 Let $(X,\|\cdot, \cdot\|)$ be a 2-normed space and consider a linearly independent subset $\left\{a_{1}, a_{2}, \ldots, a_{k}\right\}$ of $X$. Define a family of functions $\|\cdot\|_{p}$ from $X$ to $\mathbb{R}$, where $1 \leq p \leq \infty$ by

$\|x\|_{p}:=\left(\sum_{i=1}^{k}\left\|x, a_{i}\right\|^{p}\right)^{\frac{1}{p}} \quad$ for all $\quad x \in X$ when $1 \leq p<\infty$

and

$\|x\|_{p}:=\sup _{i=1,2, \ldots, k}\left\|x, a_{i}\right\|$ for all $x \in X$ when $p=\infty$.

Then, each $\|\cdot\|_{p}$ defines a norm on X. Moreover, all these norms are equivalent.

Gunawan and Mashadi [6] introduced these norms for a particular case, where $k=\operatorname{dim} X<\infty$, and called as "derived norm". As a continuation, they derived a $(n-1)$ -norm $\|\cdot, \cdot, \ldots, \cdot\|_{\infty}$ in [7] for a given $n$-normed space $X$ of arbitrary dimension. Here in Proposition 6, we have followed the same idea and rephrased derived norms for 2-normed spaces. However, the line of proof is same. Since all those derived norms are equivalent, we concentrate only on the norm $\|\cdot\|_{\infty}$.

Theorem 10 Let $(X,\|\cdot, \cdot\|)$ be a 2-normed space. Then, the following are equivalent:

I. $X$ satisfies property $\left(K_{m}\right)$ for some $m \in \mathbb{N}$,

II. there exists a linearly independent set $\left\{a_{1}, a_{2}, \ldots, a_{m}\right\}$ such that the topology of the norm defined by

$$
\begin{aligned}
& \|x\|_{\infty}:=\sup \left\{\left\|x, a_{1}\right\|,\left\|x, a_{2}\right\|, \ldots,\left\|x, a_{m}\right\|\right\} \\
& \quad \text { for all } \quad x \in X
\end{aligned}
$$$$
\text { matches with the topology of }(X,\|\cdot, \cdot\|) \text {. }
$$

Proof Let $X$ satisfies $\left(K_{m}\right)$. Then, there exists a linearly independent set $\left\{a_{1}, a_{2}, \ldots, a_{m}\right\}$ such that 
$\left\|x-x_{n}, a_{i}\right\| \rightarrow 0$ for all $i=1,2, \ldots, m$

implies $x_{n} \rightarrow x$ with respect to the 2 -norm $\|\cdot, \cdot\|$ defined on $X$.

Take the norm

$\|x\|_{\infty}:=\sup \left\{\left\|x, a_{1}\right\|,\left\|x, a_{2}\right\|, \ldots,\left\|x, a_{m}\right\|\right\}$ for all $x \in X$.

Take a sequence $\left\{x_{n}\right\}$ from $X$. Then,

$$
\begin{aligned}
& \left\|x-x_{n}, a\right\| \rightarrow 0 \text { for all } a \in X \\
& \quad \Leftrightarrow\left\|x-x_{n}, a_{i}\right\| \rightarrow 0 \text { for all } i=1,2, \ldots, m \\
& \quad \Leftrightarrow\left\|x-x_{n}\right\|_{\infty} \rightarrow 0 .
\end{aligned}
$$

So, a sequence $\left\{x_{n}\right\}$ converges in $(X,\|\cdot, \cdot\|)$ if and only if it converges in $\left(X,\|\cdot\|_{\infty}\right)$.

On the other hand, $(X,\|\cdot, \cdot\|)$ satisfies the property $\left(K_{m}\right)$ for some $m \in \mathbb{N}$. So $(X,\|\cdot, \cdot\|)$ is normable. Thus, both $(X,\|\cdot, \cdot\|)$ and $\left(X,\|\cdot\|_{\infty}\right)$ are first countable and so sequential. Therefore, both $(X,\|\cdot, \cdot\|)$ and $\left(X,\|\cdot\|_{\infty}\right)$ give the same topology.

Conversely, let both $(X,\|\cdot, \cdot\|)$ and $\left(X,\|\cdot\|_{\infty}\right)$ give the same topology where $\|\cdot\|_{\infty}$ is defined by:

$\|x\|_{\infty}:=\sup \left\{\left\|x, a_{1}\right\|,\left\|x, a_{2}\right\|, \ldots,\left\|x, a_{m}\right\|\right\}$ for all $x \in X$

for some linearly independent set $\left\{a_{1}, a_{2}, \ldots, a_{m}\right\}$.

Now let $\left\{x_{n}\right\}$ be a sequence from $X$ and $x \in X$ such that

$\left\|x-x_{n}, a_{i}\right\| \rightarrow 0$

for all $i=1,2, \ldots, m$. Then, we have $\left\|x-x_{n}\right\|_{\infty} \rightarrow 0$ and so

$\left\|x-x_{n}, a\right\| \rightarrow 0$ for all $a \in X$.

Hence, $(X,\|\cdot, \cdot\|)$ satisfies the property $\left(K_{m}\right)$.

Remark 7 Theorem 9 is useful to check the normability of a 2-normed space, and Theorem 10 is useful to construct a norm for a normable 2-normed spaces. So both those Theorems may be treated as complement of each other.

Next proposition gives a better insight for finite-dimensional 2-normed spaces.

Proposition 7 Let $(X,\|\cdot, \cdot\|)$ be a finite-dimensional 2-normed space and $a_{1}$ and $a_{2}$ be two linearly independent vectors from $X$. Then, the topology of the norm $\|\cdot\|_{\infty}$ on X defined by

$\|x\|_{\infty}:=\sup \left\{\left\|x, a_{1}\right\|,\left\|x, a_{2}\right\|\right\}$ for all $x \in X$

is the same as the topology of $(X,\|\cdot, \cdot\|)$.

Proof $X$ is finite-dimensional. Therefore, Corollary 1 concludes that $(X,\|\cdot, \cdot\|)$ is normable. Let $\|\cdot\|$ be such a norm for $(X,\|\cdot, \cdot\|)$. Now, $\|\cdot\|_{\infty}$ is also a norm on $X$. But all norms are equivalent over a finite-dimensional vector space. So $\|\cdot\|$ and $\|\cdot\|_{\infty}$ are equivalent. Hence, the topologies of $\|\cdot\|_{\infty}$ and $(X,\|\cdot, \cdot\|)$ are same.

We get two easy but important corollaries from this proposition.

Corollary 2 All 2-norms over a finite-dimensional vector space give the same topology.

Corollary 3 A finite-dimensional 2-normed space satisfies the property $\left(K_{2}\right)$.

In 2001, Gunawan and Mashadi [7] asked the following question,

Question 1 For any $n$-normed space, does there exist a derived $(n-1)$-norm such that the convergence and completeness are equivalent in both the spaces?

Using the theories developed in this paper, we give a negative answer when $n=2$.

Theorem 11 Let $(X,\|\cdot, \cdot\|)$ be a 2-normed space and $\|\cdot\|_{\infty}$ be the derived norm defined in Proposition 6. Then, convergence of sequences in $(X,\|\cdot, \cdot\|)$ and $\left(X,\|\cdot\|_{\infty}\right)$ is equivalent if and only if both the spaces have same topology.

Proof If both $(X,\|\cdot, \cdot\|)$ and $\left(X,\|\cdot\|_{\infty}\right)$ have same topology, then obviously convergence of sequences is equivalent. Conversely, let convergence of sequences for $(X,\|\cdot, \cdot\|)$ and $\left(X,\|\cdot\|_{\infty}\right)$ is equivalent. Let $\left\{x_{n}\right\}$ be any sequence in $X$ and $x \in X$. Then,

$$
\begin{aligned}
& x_{n} \rightarrow x \operatorname{in}(X,\|\cdot, \cdot\|) \Longleftrightarrow x_{n} \rightarrow x \text { in }(X,\|\cdot\|) \\
& \quad \text { i.e. } \lim _{n \rightarrow \infty}\left\|x-x_{n}, a\right\|=0 \quad \forall a \in X \Longleftrightarrow \lim _{n \rightarrow \infty}\left\|x-x_{n}\right\|_{\infty}=0 .
\end{aligned}
$$

However, the definition of $\|\cdot\|_{\infty}$ concludes the existence of a linearly independent set $\left\{a_{1}, a_{2}, \ldots, a_{m}\right\}$ such that for any sequence $\left\{x_{n}\right\}$ in $X$ and $x \in X$ the following holds,

$\lim _{n \rightarrow \infty}\left\|x-x_{n}\right\|_{\infty}=0 \Longleftrightarrow \lim _{n \rightarrow \infty}\left\|x-x_{n}, a_{i}\right\|=0 \quad \forall i=1,2, \ldots, m$.

Combining Eqs. (5) and (6), we get, for any sequence $\left\{x_{n}\right\}$ in $X$ and $x \in X$

$$
\begin{aligned}
& \lim _{n \rightarrow \infty}\left\|x-x_{n}, a\right\|=0 \quad \forall a \in X \\
& \quad \Longleftrightarrow \lim _{n \rightarrow \infty}\left\|x-x_{n}, a_{i}\right\|=0 \quad \forall i=1,2, \ldots, m .
\end{aligned}
$$


Therefore, $(X,\|\cdot, \cdot\|)$ satisfies property $\left(K_{m}\right)$. Thus, using Theorem 10 , we can say that $(X,\|\cdot, \cdot\|)$ and $\left(X,\|\cdot\|_{\infty}\right)$ have same topology.

Here is an example related to Theorem 11.

Example 2 Let $X=l^{\infty}$, the space of all bounded real sequences. Define a 2-norm on $X$ by

$\|x, y\|:=\sup _{i \in \mathbb{N}} \sup _{j \in \mathbb{N}}\left|x_{i} y_{j}-x_{j} y_{i}\right|$

where $x=\left(x_{1}, x_{2}, x_{3}, \ldots\right)$ and $y=\left(y_{1}, y_{2}, y_{3}, \ldots\right)$. Gunawan and Mashadi [7] showed that for $a_{1}=(1,0,0, \ldots)$ and $a_{2}=(0,1,0,0, \ldots)$ in $X$, the derived norm $\|\cdot\|_{\infty}$ on $X$ defined by

$\|x\|_{\infty}:=\sup \left\{\left\|x, a_{1}\right\|,\left\|x, a_{2}\right\|\right\}$

satisfies the following condition: for sequence $\left\{x_{n}\right\}$ in $X$ and $x \in X, x_{n} \rightarrow x$ in $(X,\|\cdot, \cdot\|)$ if and only if the same happens in $\left(X,\|\cdot\|_{\infty}\right)$. Now Theorem 11 allows us to conclude that both $(X,\|\cdot, \cdot\|)$ and $\left(X,\|\cdot\|_{\infty}\right)$ have same topology, i.e. $(X,\|\cdot, \cdot\|)$ is normable. In particular, it is metrizable. But $X=l^{\infty}$ has uncountable Hamel basis. So the converse of Theorem 6 is not true.

To answer Question 1, we summarize Theorems 9-11 as follows:

Theorem 12 Let $(X,\|\cdot, \cdot\|)$ be a 2-normed space and $\|\cdot\|_{\infty}$ be the derived norm defined in Proposition 6. Then, the following are equivalent,

(i) convergence of sequences in $(X,\|\cdot, \cdot\|)$ and $\left(X,\|\cdot\|_{\infty}\right)$ are equivalent,

(ii) $(X,\|\cdot, \cdot\|)$ and $\left(X,\|\cdot\|_{\infty}\right)$ have the same topology,

(iii) $(X,\|\cdot, \cdot\|)$ satisfies property $\left(K_{m}\right)$ for some $m \in \mathbb{N}$,

(iv) $(X,\|\cdot, \cdot\|)$ is normable.

This clearly indicates that convergence of sequences with respect to a 2-norm and its derived norm are not equivalent if the 2-normed space is not normable. However, Example
1 assures the existence of 2-normed spaces which are not normable. Hence, we get a negative answer to Question 1.

Acknowledgements The authors are grateful to the anonymous referee for his/her valuable suggestions and comments in rewriting the article in the present form. The authors are also thankful to the Editor-inChief and the Associate Editor of the journal (Mathematical Sciences) for their valuable comments. The research of first author is funded by UGC, New Delhi, India, under NET-JRF scheme (Sr. No. 2061441028, Ref. No.: 22/06/2014(i)EU-V). The research of second author is partially supported by the Special Assistance Programme (SAP) of UGC, New Delhi, India [Grant NO. F 510/3/DRS-III/ 2015 (SAP-I)].

Open Access This article is distributed under the terms of the Creative Commons Attribution 4.0 International License (http://creativeco mmons.org/licenses/by/4.0/), which permits unrestricted use, distribution, and reproduction in any medium, provided you give appropriate credit to the original author(s) and the source, provide a link to the Creative Commons license, and indicate if changes were made.

\section{References}

1. Gähler, S.: 2-metrische räume und ihre topologische struktur. Math. Nachr. 26(1-4), 115-148 (1963)

2. Gähler, S.: Lineare 2-normierte räume. Math. Nachr. 28(1-2), $1-43(1964)$

3. Gähler, S.: Untersuchungen über verallgemeinerte m-metrische räume. I. Math. Nachr. 40(1-3), 165-189 (1969)

4. Gähler, S.: Untersuchungen über verallgemeinerte m-metrische räume. II. Math. Nachr 40(4-6), 229-264 (1969)

5. Gähler, S.: Untersuchungen über verallgemeinerte m-metrische räume. III. Math. Nachr. 41(1-3), 23-36 (1969)

6. Gunawan, H., Mashadi, : On finite dimensional 2-normed spaces. Soochow J. Math. 27(3), 321-329 (2001)

7. Gunawan, H., Mashadi, M.: On $n$-normed spaces. Int. J. Math. Math. Sci. 27(10), 631-639 (2001)

8. Heil, C.: Five Courses on Analysis. Birkhäuser, Boston (to appear)

9. Rudin, W.: Functional Analysis. International Series in Pure and Applied Mathematics. McGraw-Hill Inc, New York (1991)

10. Vulich, B.: On a generalized notion of convergence in a Banach space. Ann. Math. 38(1), 156-174 (1937)

11. Zimmer, R.J.: Essential Results of Functional Analysis. University of Chicago Press, Chicago (1990)

Publisher's Note Springer Nature remains neutral with regard to jurisdictional claims in published maps and institutional affiliations. 